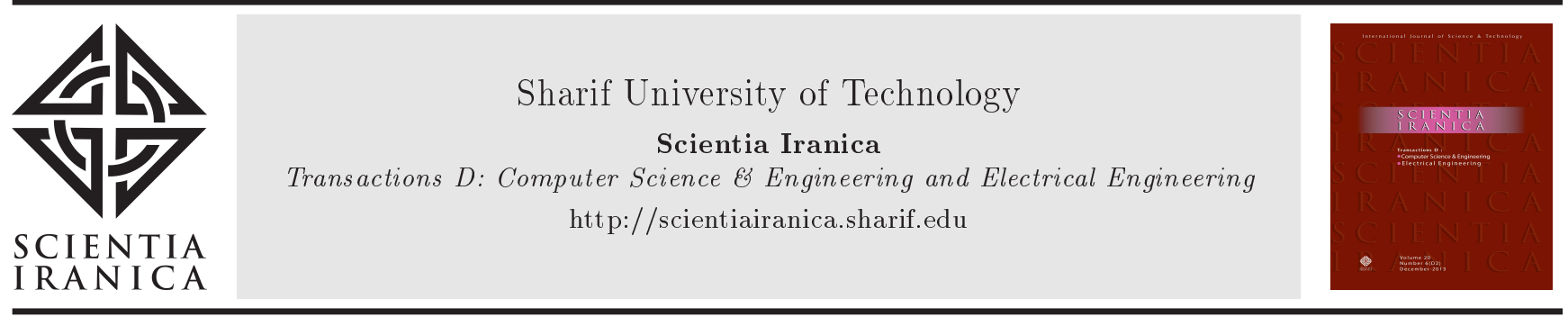

\title{
Design optimization of a low-speed small-scale modular axial flux permanent magnet synchronous generator for urban wind turbine application
}

\author{
H. Saneie ${ }^{\mathrm{a}}$, A. Daniar ${ }^{\mathrm{b}}$, and Z. Nasiri-Gheidari ${ }^{\mathrm{a}, *}$ \\ a. Department of Electrical Engineering, Sharif University of Technology, Tehran, Iran. \\ b. Department of Electrical and Computer Engineering, The University of Texas at Dallas, Richardson, TX, USA. \\ Received 13 July 2020; received in revised form 17 November 2020; accepted 4 January 2021
}

\author{
KEYWORDS \\ Wind turbine; \\ Clean energy; \\ Permanent Magnet \\ Synchronous Motor \\ (PMSM); \\ Axial flux; \\ Analytical model; \\ Optimization; \\ Finite Element \\ Method (FEM)
}

\begin{abstract}
Small wind turbines can generate clean energy in diverse locations, from urban to rural areas, without access to the main grids. This study proposes design optimization of an Axial Flux Permanent Magnet (AFPM) synchronous machine for small-scale portable applications. At first, a fast, accurate and pragmatic hybrid analytical model is proposed for performance prediction of the machine. The proposed model is then used as a basis for direct search optimization with factual constraints and objective functions to improve the machine in terms of efficiency, weight, and convenience of the manufacturing process. 3Dimensional Time Stepping Finite Element Method (3-DTSFEM) is utilized to validate the accuracy and effectiveness of the proposed model and optimization methodology. Output characteristics of the machine from both analytical model and 3-DTSFEM are compared to each other, thus proving the functionality of the proposed study.

(C) 2022 Sharif University of Technology. All rights reserved.
\end{abstract}

\section{Introduction}

Recently, wind energy has attracted much attention as a clean energy to replace fossil fuels since it is a readily and naturally available source. It was anticipated that by 2021 , more than $1000 \mathrm{GW}$ of the power in the world was produced by wind energy [1]. In future, urban small wind turbines will play a massive role along with giant ones in producing a massive amount of power. In this regard, there is a growing interest to use smallscale wind turbines in buildings. Thus, it is essential to research the electric generator, which is a crucial part of wind energy conversion systems. Commonly, wind speed in urban areas is low and one good choice for such applications is Axial Flux Permant Magnet

*. Corresponding author. Tel.: +982166164389 E-mail address: znasiri@sharif.edu (Z. Nasiri-Gheidari)

doi: $10.24200 /$ sci.2021.56405.4709
(AFPM) generator with a large number of poles. An AFPM generator enjoys high power density and high efficiency. Since this type of machine has a small axial length, it can be used in an axially modular structure to reach higher output power [2-8]. Numerous studies have been conducted in this domain [9-11].

Multi-objective design optimization of a yokeless and segmented armature (YASA machine) for direct drive wind turbine has been investigated. Pareto front method is utilized to find the best configuration for the poles and stator segments in terms of minimizing the inherent cogging torque of the machine [12]. A doublestator and three-rotor AFPM synchronous machine is designed so as to reduce iron loss and weight of the machine for direct drive applications [13]. Hijazi et al. [14] evaluated the comparative stator design of armature copper conductors with eddy current loss and AFPM generators with stator core losses for wind turbine application. A new type of Permanent Magnet (PM) machine having both radial and axial flux, so- 
called hybrid-flux, was presented in [15]. The power density of the proposed generator was 9.4 times larger than that of the commercialized one. In case winding consists of non-overlapping concentrated coils and has a non-integral coil-pole ratio, with an appropriate choice of armature coil number to pole number, the fundamental winding factor of the AFPM synchronous generator can be made close to that of a full-pitched integral slot winding structure [16]. A novel technique was employed for modeling Partitioned Stator Flux-Switching PM (PS-FSPM) machines by Magnetic Equivalent Circuit (MEC), which considers saturation effects by nonlinear reluctances and provides adjustable accuracy for modeling the machine [17]. Widyan and Hanitsch [18] presented a new three-slot threephase four-pole rare-earth radial-flux PM variablespeed electrical generator with soft magnetic material. Some studies have studied synchronous machines with Halbach array of PMs. An appropriate configuration of Halbach PMs was extracted and then, a Nondominated Sorting Genetic Algorithm-II (NSGA-II) technique was used to optimize the efficiency, size, and mass of the generator. The winding of the machine is Printed Circuit Board (PCB) based [19].

Design optimization of an electrical machine requires a mathematical model for performance prediction and analysis of the machine. Typically, Finite Element Analysis (FEA) is the most accurate approach to analyzing an electric machine; however, due to 3Dimensional (3-D) structure of AFPM generator, it is almost impossible to run an optimization loop based on the Finite Element Method (FEM) because it takes much time to do the process.

Use of empirical data (e.g., sizing equations) is another way that significantly shortens simulation time and can be used as a basis for optimization processes [20]. However, this method is not quite accurate due to poor estimation of equivalent circuit parameters. Different analytical models including Winding Function (WF) [21,22], MEC [23], and Fourier series method [24] are developed for designing and optimization of electrical machines. An analytical model enjoys shorter simulation time and comparable accuracy, compared to FEM. An enhanced model of MEC model for evaluation of air-gap leakage flux of AFPM generator was presented in [25]. AlipourSarabi et al. [26] applied a general analytical modeling technique to evaluate the performance of axial flux machines. The proposed model is based on the actual three-dimensional MEC. Flux fringing effect, skewed slots, static and dynamic eccentricity, and core magnetization curve are included in the model to make it as accurate as possible. Multi-objective optimization design of air-cored AFPM generators based on TaguchiResponse Surface Method (RSM) was proposed in [27]. The main characteristics of generator efficiency, power quality, induction electromotive force, and others are taken as optimization objectives.

In this paper, design and optimization of a coreless surface mounted double-rotor AFPM generator is presented for low-speed wind turbine applications. At first, design considerations and practical limitations are expressed to determine geometrical parameters including the shape of PMs and length of back iron. Furthermore, the relationship between different dimensional parameters was obtained to reduce the design and optimization variables. Thereafter, a very fast and accurate analytical model was presented through direct solving of Maxwell equations and combining with the classical model of synchronous generators. In the developed model, 3D effects such as end winding of stators' coil and variation of geometrical parameters in $r$-component (along with radius) are considered. The hybrid analytical model is used to design a 500 Watts generator. Then, the optimization model is applied to promote the efficiency of the designed generator and reduce the material cost and weight. Due to the very short simulation time of the model at each iteration, the entire optimization process takes less than $15 \mathrm{~min}$. Finally, all analyses are verified by 3 -Dimensional Time Stepping Finite Element Method (3-DTSFEM). It is shown that the optimal design reduces the PM weight and total weight by $22.5 \%$ and $11.1 \%$, respectively, and the efficiency of optimal design increases by $2.5 \%$.

\section{Machine structure and design consideration}

In Figure 1(a), the stator and rotors of a 3-phase AFPM generator are shown. As can be seen, the stator is composed of a 3-phase winding, which is located between two rotors. Each stator winding consists of a number of similar coils placed in sequential patterns. It should be noted that there is no stator core in this configuration; therefore, the windings are located on a non-ferromagnetic material.

Each rotor includes a non-laminated ferromagnetic disk with $N_{p m}$ surface-mounted trapezoidal PMs

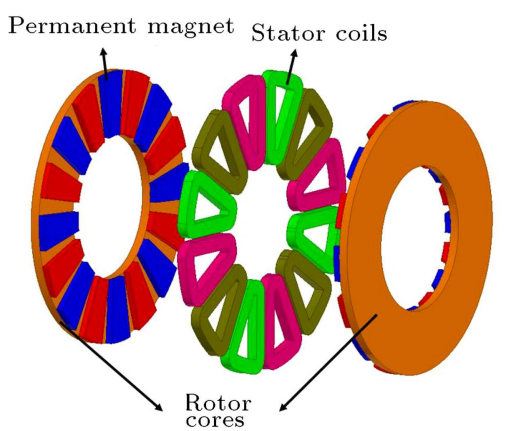

(a)

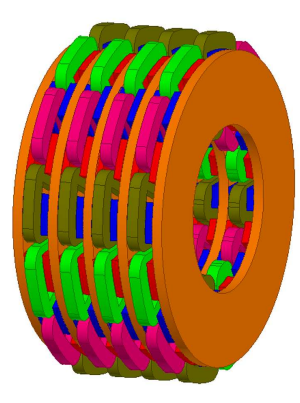

(b)
Figure 1. Structure of the coreless AFPM generator: (a) Exploded view of one module and (b) illustration of four module. 
such that their polarization direction varies from one PM to another. In general, the relationship between stator coils and rotor PMs is followed by:

$$
\frac{N_{c}}{N_{p m}}=\frac{m}{m+1},
$$

where $m$ is number of phases, $N_{c}$ is number of stator coils, and $N_{p m}$ is number of PMs on each rotor or number of poles.

In this machine, the magnetic field in rotor cores is not alternative; therefore, rotor core loss is negligible. As a result, rotor core can be made by non-laminated materials. Moreover, the stator is air cored and has no magnetic material; accordingly, the overall core loss in this generator is negligible.

As mentioned before, axial flux machines have an inherently more efficient topology in electromagnetic sense. In addition, due to small axial length and special structure of axial flux machines, they can be used in modular structure by putting them together (Figure 1(b)). Moreover, in such configurations, to reduce stack length and weight of the machine further instead of using two rotor cores for each module, it is possible to mount PMs on both sides of each rotor core. However, one must keep in mind that the thickness of the rotor core should be calculated until there is no saturation.

Therefore, to obtain a certain level of output power, the number of modules may vary. In general, the output diameter of the axial flux machine is too large compared to its radial counterpart for the same power output, especially in coreless types. Therefore, upon using a modular structure, one can reduce the power of each module by reducing the outer diameter and using additional modules to obtain the desirable output power. In this way, there is a balance between radial and axial lengths of the whole system, making it more rigid and robust against radial and axial forces.

\subsection{PMs shape}

As shown in Figure 1(a), PMs are trapezoidal because PM volume, or PM energy, has a direct relation with the output power of the machine. Compared to other PM shapes, the trapezoidal shape provides maximum volume at constant thickness of the PM and constant inner and outer diameters of the rotor. Moreover, trapezoidal PMs are not cost-efficient and are, also, not widely accessible in the market. As can be seen in Figure 2, instead of using one trapezoidal PM, two rectangular PMs with different widths and lengths can be used. In this structure, in order to maximize the volume of the PMs, it is necessary that the width of the inner PM $\left(W_{p m 1}\right)$ be maximum. With the inner diameter of the rotor and number of the poles at hand, the width of the inner PM can be calculated by using Eq. (2):

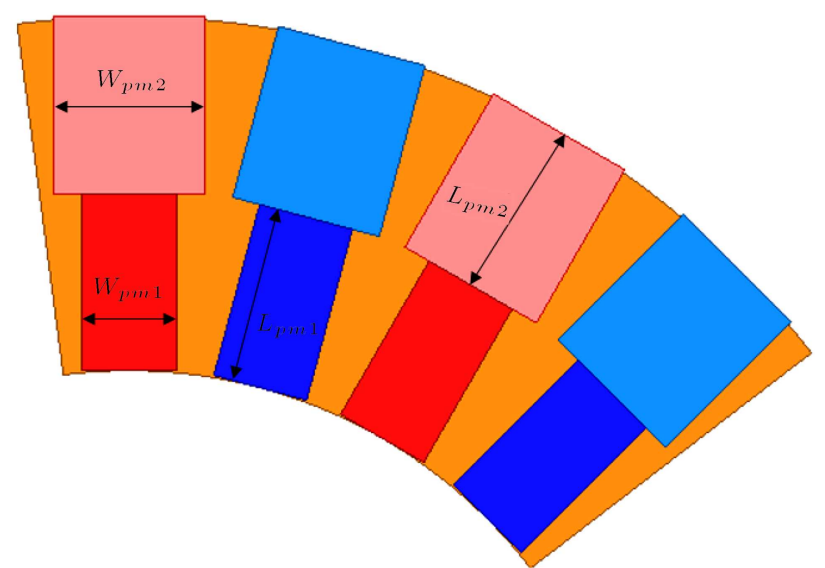

Figure 2. Illustration of using two rectangular PMs instead of one trapezoidal PMs.

$$
W_{p m 1}=D_{i} \tan ^{-1} \frac{\pi}{N_{p m}},
$$

where $W_{p m 1}$ is the width of the inner PM in mm, $D_{i}$ the inner diameter of the rotor in $\mathrm{mm}$, and $N_{p}$ the number of poles.

In order to make the design of the PMs compatible with the availability of PMs in the market, the standard size of the PM width is changing with $1 \mathrm{~mm}$ steps. Hence, by using the same procedure and taking the length of the inner PM $\left(L_{p m 1}\right)$ into account, the width of the outer PM $\left(W_{p m 2}\right)$ is then calculated.

Likewise, if there are more rectangular PMs, the width of each PM can be calculated with the same procedure. Moreover, it can be proved that when using two rectangular PMs instead of one trapezoidal PM, the total cross-section of the PMs is maximum when they have the same length, $L_{p m 2}=L_{p m 1}$.

\subsection{Coils}

Figure 3 shows one of the coils of stator winding. Since core loss for this machine is negligible, the majority of power loss originates from the copper loss in the winding, as mentioned before. At constant output power, the cross-section of the coils is larger, copper

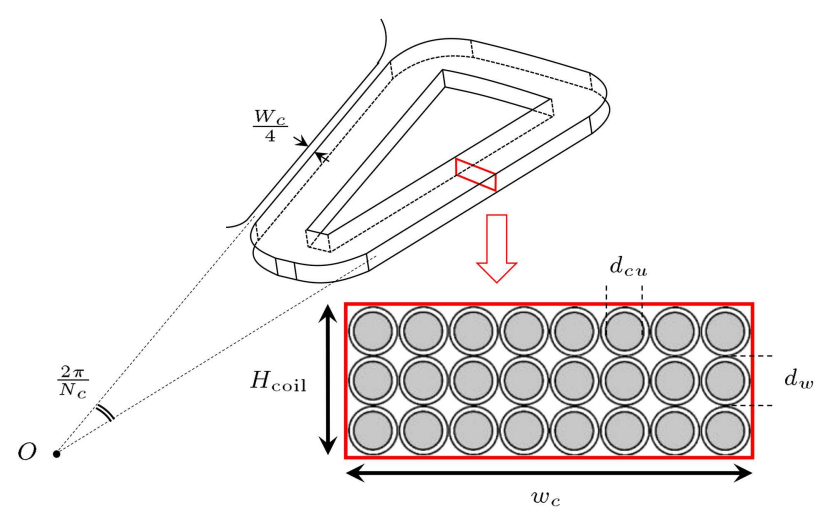

Figure 3. Structure of a stator's coil. 
loss lower, and the efficiency of the machine higher. As a result, the cross-section of the coils should be at their maximum possible value.

In order to increase the cross-sectional area of the coils, either width $\left(W_{c}\right)$ or height $\left(H_{\text {coil }}\right)$ must be increased. Width of the coils is determined based on the inner diameter of the machine and number of the coils. Since these two parameters are already known, the maximum width of the coils can be calculated based on Eq. (1). As mentioned before, another way to increase the cross-sectional area of the coils is to increase their height. Unlike the width which does not significantly change the behavior of the machine, when the height of the coil increases, the air-gap length is basically increasing, which affects the whole characteristic of the machine. Thus, to have the freedom of increasing the height of the coil, this parameter should be effectively considered in the model so that it can predict the power, efficiency, etc. for different heights of the coil.

With the width and height of the coils at hand, the number of conductors per coils $\left(T_{c}\right)$ is roughly calculated (Figure 3 ). Moreover, since the diameter of the conductor has been determined $\left(d_{c u}\right)$, filling factor $\left(f_{c u}\right)$ can be calculated, too [28]:

$$
f_{c u}=\frac{T_{c} A_{c u}}{A_{\text {slot }}}=\frac{T_{c} \pi d_{c u}^{2}}{4 W_{c} H_{c o i l}} .
$$

Resistance of the coils $\left(R_{c}\right)$ is then calculated as follows (Figure 3):

$$
R_{c}=\frac{L_{c u}}{\sigma A_{c u}},
$$

where $\sigma$ electrical conductivity of coil; $A_{c u}$ and $L_{c u}$ are the area and equivalent length of each coil, respectively, which are calculated as:

$$
\begin{aligned}
A_{c u} & =\frac{\pi d_{c u}^{2}}{4}=\frac{W_{c} H_{c o i l}}{T_{c}} f_{c u} \\
L_{c u} & =T_{c}\left((\pi-4.5) W_{c}+\frac{\pi}{N_{c}}\left(D_{o}+D_{i}\right)+\left(D_{o}-D_{i}\right)\right) .
\end{aligned}
$$

Then, the resistance of each phase is calculated as follows:

$$
R_{\text {phase }}=\frac{R_{c} N_{c}}{m}
$$

This will be used to calculate $\mathrm{I}^{2} \mathrm{R}$ losses and, finally, the efficiency of the machine.

Same as PMs, to design coils, the diameter of the conductors is considered in discrete steps to make it feasible and compatible with the actual manufacturing process. For this matter, the diameter of the conductor is changed from 0.2 to $1 \mathrm{~mm}$ with 0.05 steps and the increasing step is $0.1 \mathrm{~mm}$ after $1 \mathrm{~mm}$.
As discussed earlier, the main objective of this paper is to design a coreless modular AFPM machine first with the maximum of 500 Watts for each module and then, to optimize the machine to ensure maximum efficiency and minimize the weight.

It is quite well known that for designing any complicated device such as an electric machine, it is necessary to have a mathematical tool to predict the behavior of that device. In this regard, FEA is one of the numerical methods that is very popular and probably the most accurate way to solve mechanical, thermal, and electromagnetic problems. FEA is a perfect tool to design the initial sample. However, in the case of optimization, FEA may not be quite a viable option. The reason is that most optimization algorithms work based on iterations and usually take thousands of iterations to reach the most optimum results. All in all, the need for an accurate and fast model for analyzing and predicting the behavior of the machine in different conditions and with different parameters is irrefutable.

\section{The analytical model}

Different analytical models including MEC [23] and WF models [21] have been proposed for analyzing electric machines. Compared with FEA, these models are not accurate enough because both MEC and WF consider only the normal component of the field. In Permanent Magnet Synchronous Motors (PMSMs), due to the longer air-gap length, the tangential component of the field has a significant impact that should be taken into account [29]. Despite these models, the methods that are based on solving Maxwell equations can accurately calculate the $2 \mathrm{D}$ field, leading to a good prediction of leakage and fringing flux of the PMs. Maxwell equation-based models are subject to their own deficiencies, too. For instance, for electric machines with teeth and slots, the matter gets very complicated and machine designers try to compensate that effect with particular coefficients like Carter's coefficient, decreasing the accuracy of the model.

In this section, a $2 \mathrm{D}$ analytical model for the mentioned Permanent Magnet Synchronous Generator (PMSG) based on the direct solving of Maxwell equation is presented. For the sake of simplicity, the following assumptions have been considered:

- Magnetic permeability of ferromagnetic is infinite $\left(\mu_{\text {iron }}=\infty\right)$;

- PMs magnetic permeability is equal to air gap $\left(\mu_{p m}=\mu_{0}\right)$

- The $r$-component of magnetic field is zero (radial edge effect neglected);

- Curvature effect on the axial flux structure is neglected. 


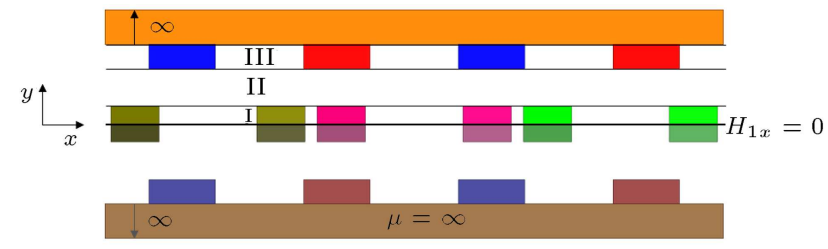

(a)

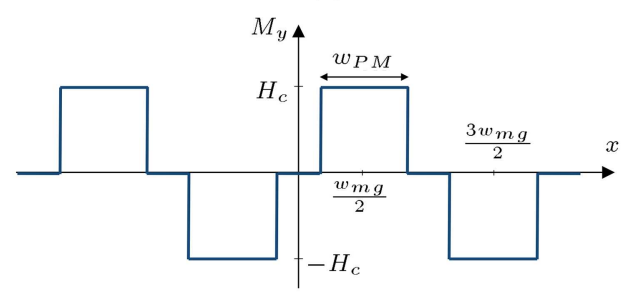

(b)

Figure 4. Analytical model: (a) cross-section of machine in specified radius and (b) magnetic polarization of PMs.

Figure 4(a) shows the cross-section of the machine at a specified radius. Based on the machine structure, it can be concluded that the magnetic field in upper and lower halves of the machine is symmetric and $x$ component of the magnetic field on $y=0$ axis is equal to zero (Figure $4(\mathrm{a})$ ). Therefore, it is not only accurate but also faster to perform the calculations for only one half of the machine. Vector potential in different layers of the machine is calculated by solving Poisson's equation [24]:

$$
\nabla^{2} A_{z}=-\mu_{0}\left(\nabla \times M_{0}\right)-\mu_{0} J_{z},
$$

where $M_{0}$ is residual magnetization, $J_{z}$ current density, $A_{z}$ magnetic vector potential, and $\mu_{0}$ the permeability of free space. In the following, $A_{z}$ created by PM and coils' currents are calculated separately. Based on superposition theorem, it is a valid and straightforward way of calculating vector potentials.

\subsection{Permanent Magnets (PMs)}

Figure 4(b) shows the magnetic polarization distribution of PMs. Fourier series of magnetic Polarization is calculated as:

$$
M_{y}=\sum_{n=1,3,5, \cdots} b_{P M n} \sin \left(n \omega_{P M} x\right),
$$

where $n$ is harmonic order; $M_{y}$ is magnetic polarization in the $y$ direction; $\omega_{P M}$ and $b_{P M n}$ are:

$$
\begin{aligned}
& \omega_{P M}=\frac{N_{p m}}{2 r}, \\
& b_{P M n}=\frac{4 H_{c}}{n \pi} \sin \frac{n \omega_{\mathrm{PM}} w_{m g}}{2} \sin \frac{n \omega_{P M} w_{P M}}{2},
\end{aligned}
$$

where $w_{P M}$ is the width of PM, $H_{c}$ the coercivity of PMs, and $w_{m g}$ calculated as follows:

$$
w_{m g}=\frac{2 \pi}{N_{p m}} r
$$

where $r$ is the specific radius of the machine.
Irrespective of the current density of coils, coil area (region I) and air gap area (region II) are the same. Therefore, by solving Eq. (8), the magnetic vector potential in regions I and II $\left(A_{\mathrm{I}-\mathrm{II}, Z}^{P M}\right)$ is calculated as follows:

$$
\begin{aligned}
A_{\mathrm{I}-\mathrm{II}, Z}^{P M}= & \sum_{n=1,3, \cdots}^{\infty}\left[C_{1 n}^{P M} e^{n \omega_{P M} y}+D_{1 n}^{P M} e^{-n \omega_{P M} y}\right] \\
& \cos \left(n \omega_{P M} x\right),
\end{aligned}
$$

and magnetic vector potential in region III $\left(A_{\mathrm{III}, Z}^{P M}\right)$ is obtained by:

$$
\begin{aligned}
A_{\mathrm{III}, Z}^{P M}= & \sum_{n=1,3, \cdots}^{\infty}\left[C_{3 n}^{P M} e^{n \omega_{P M} y}+D_{3 n}^{P M} e^{-n \omega_{P M} y}\right. \\
& \left.+K_{P M}(n)\right] \cos \left(n \omega_{P M} x\right)
\end{aligned}
$$

where constant coefficients, $C_{1 n}^{P M}, C_{3 n}^{P M}, D_{1 n}^{P M}, D_{3 n}^{P M}$ and particular solution of Eq. (8), $K_{P M}$, are calculated under boundary conditions:

$$
\begin{aligned}
& C_{1 n}^{P M}=\frac{0.5 \times K_{P M}(n)}{\cosh \left(n \omega_{P M} h_{1}\right)+\frac{\sinh \left(n \omega_{P M} h_{1}\right)}{\tanh \left(n \omega_{P M} H_{P M}\right)}}, \\
& C_{3 n}^{P M}=-e^{n \omega_{P M} h_{2}} \frac{\sinh \left(n \omega_{P M} h_{1}\right)}{\sinh \left(n \omega_{P M} H_{P M}\right)} C_{1 n}^{P M}, \\
& D_{1 n}^{P M}=C_{1 n}^{P M}, \\
& D_{3 n}^{P M}=e^{2 n \omega_{P M} h_{2}} C_{3 n}^{P M}, \\
& K_{P M}(n)=\frac{\mu_{0} b_{P M n}}{n \omega_{P M}},
\end{aligned}
$$

where $H_{P M}$ is the height of PMs and $h_{1}, h_{2}$ are:

$$
\begin{aligned}
& h_{1}=\frac{H_{\text {coil }}}{2}+g, \\
& h_{2}=h_{1}+H_{P M},
\end{aligned}
$$

where $H_{\text {coil }}$ and $g$ are the height of coils and air gap length, respectively.

\subsection{Stator coils}

To calculate magnetic vector potential of stator coils, the Fourier series of coils current must be obtained in the first step. Figure 5 shows the current density for phase $b$. Therefore, we have:

$$
J_{W b}=\sum_{n=1,2,3, \cdots} b_{n b} \sin \left(n \omega_{w} x\right)
$$

where $J_{w b}$ is the current density of phase $b$ and $\omega_{w}$ and $b_{n b}$ are: 


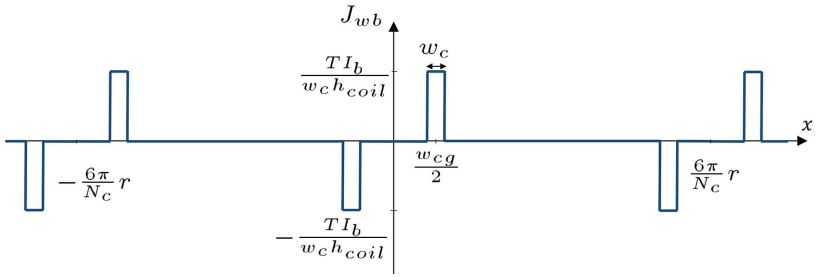

Figure 5. Current density distribution of stator coils (phase $b)$.

$$
\begin{aligned}
\omega_{w} & =\frac{N_{c}}{3 r}, \\
b_{n b} & =\frac{4 J_{b m}}{n \pi} \sin \frac{n \omega_{w} w_{c g}}{2} \sin \frac{n \omega_{w} w_{c}}{2}, \\
J_{b m} & =\frac{T I_{b}}{w_{c} H_{c o i l}},
\end{aligned}
$$

where $T$ is the number of turns in each coil of stator, $I_{b}$ current of phase $b, w_{c}$ the coil width, and $w_{c g}$ is as follows:

$$
w_{c g}=\frac{2 \pi}{N_{c}} r-1.25 w_{c} .
$$

Since PMs are neglected, regions II and III are the same. Therefore, $A_{z}$ in region I is calculated as follows:

$$
\begin{aligned}
A_{\mathrm{I}, Z}^{\text {coil }}= & \sum_{n=1,2,3, \cdots}^{\infty}\left[C_{1 n}^{\text {coil }} e^{n \omega_{w} y}+D_{1 n}^{c o i l} e^{-n \omega_{w} y}\right. \\
& \left.+K_{w b}(n)\right] \sin \left(n \omega_{w} x\right) .
\end{aligned}
$$

Moreover, in regions II and III, we have:

$$
\begin{aligned}
A_{\mathrm{II}-\mathrm{III}, Z}^{\text {coil }}= & \sum_{n=1,2,3, \cdots}^{\infty}\left[C_{3 n}^{\text {coil }} e^{n \omega_{w} y}+D_{3 n}^{\text {coil }} e^{-n \omega_{w} y}\right] \\
& \sin \left(n \omega_{w} x\right),
\end{aligned}
$$

where constant coefficients, $C_{1 n}^{\text {coil }}, C_{3 n}^{\text {coil }}, D_{1 n}^{\text {coil }}, D_{3 n}^{\text {coil }}$, and the particular solution of Eq. (8), $K_{w b}$, are calculated by applying boundary conditions:

$$
\begin{aligned}
& C_{1 n}^{c o i l}=\frac{-0.5 \times K_{w b}(n)}{\cosh \left(\frac{n \omega_{w} H_{\text {coil }}}{2}\right)+\frac{\sinh \left(\frac{n \omega_{w} H_{\text {coil }}}{2}\right)}{\tanh \left(n \omega_{w}\left(h_{P M}+g\right)\right)}}, \\
& C_{3 n}^{c o i l}=-e^{-n \omega_{w} h_{2}} \frac{\sinh \left(\frac{n \omega_{w} H_{c o i l}}{2}\right)}{\sinh \left(n \omega_{w}\left(h_{P M}+g\right)\right)} C_{1 n}^{c o i l}, \\
& D_{1 n}^{c o i l}=C_{1 n}^{c o i l}, \\
& D_{3 n}^{c o i l}=e^{2 n \omega_{w} h_{2}} C_{3 n}^{c o i l}, \\
& K_{w b}(n)=\frac{\mu_{0} b_{n b}}{\left(n \omega_{w}\right)^{2}} .
\end{aligned}
$$

To calculate the magnetic vector potential for phase $a$ and $c$, it is enough to make a 120-degree phase shift in Eqs. (27) and (28).

\subsection{Consideration of $3 D$ effect}

So far, the 2D magnetic field created by PMs and phase current has been calculated in a specific diameter. In this way, one can compute the total magnetic field based on superposition theorem and by integrating the $2 \mathrm{D}$ magnetic field throughout the radial axis of the machine. To make the model more accurate and consistent with 3D structure of the machine, some effects like the change in PMs' width and the location of the coils at different radii should be taken into account. Since there are two different widths for the PMs, the machine is divided into two regions, $A$ and $B$, as shown in Figure 6. Magnetic fields in each region are calculated separately; then, they are summed up together. To ensure even higher accuracy, one can increase the number of regions.

\subsection{Flux linkage}

To obtain output voltage, self and mutual inductances, linkage flux due to PMs, and phase currents should be calculated. Flux linkage for each coil is calculated as follows:

$$
\varphi=\int \vec{B} \cdot d \vec{s}=l\left(A_{1}-A_{2}\right)
$$

where $l$ is the axial length of the region where flux linkage is calculated. $A_{1}$ and $A_{2}$ are the average values of vector potentials of each leg of the coil. To clarify, $A_{1}$ and $A_{2}$ created by PMs for the coil related to phase $b\left(A_{1, b}^{P M}\right.$ and $\left.A_{2, b}^{P M}\right)$, shown in Figure $4(\mathrm{a})$, are obtained as follows:

$$
\begin{aligned}
A_{1, b}^{P M} & =\sum_{n=1,3, \cdots}^{\infty} \mathcal{C}_{c o i l, n}^{P M} \times \cos \left(n \omega_{P M}\left(\frac{w_{c g}}{2}+\chi\right)\right) \\
A_{2, b}^{P M} & =\sum_{n=1,3, \cdots}^{\infty} \mathcal{C}_{\text {coil }, n}^{P M} \times \cos \left(n \omega_{P M}\left(\frac{w_{c g}}{2}-\chi\right)\right)
\end{aligned}
$$

where $\chi$ is the position of the rotor and $\mathcal{C}_{\text {coil }, n}^{P M}$ can be calculated from the equation below:

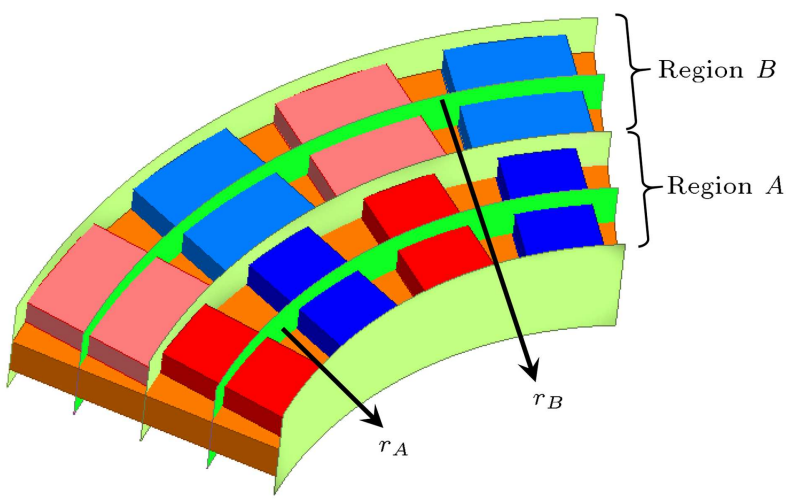

Figure 6. Consideration of radial change in the model. 


$$
\begin{aligned}
\mathcal{C}_{\text {coil }, n}^{P M}= & \frac{8 C_{1 n}^{P M}}{W_{c} H_{\text {coil }}\left(n \omega_{P M}\right)^{2}} \sinh \left(\frac{n \omega_{P M} H_{\text {coil }}}{2}\right) \\
& \sin \left(\frac{n \omega_{P M} w_{c}}{2}\right) .
\end{aligned}
$$

Vector potentials for self-linkage flux of each coil $\left(A_{1}^{\text {self }}\right.$ and $A_{2}^{\text {self }}$ ) are calculated as follows:

$$
A_{1}^{\text {self }}=-A_{2}^{\text {self }}=\sum_{n=1,2,3, \cdots}^{\infty} \mathcal{C}_{\text {coil }, n} \sin \left(\frac{n \omega_{w} w_{c g}}{2}\right)
$$

Moreover, vector potentials for mutual-linkage flux of the adjacent coils $\left(A_{1}^{\text {mutual }}\right.$ and $\left.A_{2}^{\text {mutual }}\right)$ can also be calculated as follows:

$$
\begin{aligned}
& A_{1}^{\text {mutual }}=\sum_{n=1,2,3, \cdots}^{\infty} \mathcal{C}_{\text {coil }, n} \sin n\left(\frac{\omega_{w} w_{c g}}{2}-\frac{2 \pi}{3}\right) \\
& A_{2}^{\text {mutual }}=-\sum_{n=1,2,3, \cdots}^{\infty} \mathcal{C}_{\text {coil }, n} \sin n\left(\frac{\omega_{w} w_{c g}}{2}+\frac{2 \pi}{3}\right),
\end{aligned}
$$

where $\mathcal{C}_{\text {coil }, n}$ is:

$$
\begin{aligned}
\mathcal{C}_{\text {coil }, n}= & \frac{4}{W_{c} H_{c o i l}\left(n \omega_{w}\right)^{2}}\left\{2 C_{1 n}^{\text {coil }} \sinh \left(\frac{n \omega_{w} H_{\text {coil }}}{2}\right)\right. \\
& \left.+\frac{n \omega_{w} H_{c o i l} K_{w b}(n)}{2}\right\} \sin \left(\frac{n \omega_{w} w_{c}}{2}\right)
\end{aligned}
$$

\subsection{Output characteristics}

In general, to obtain the full output characteristics of the machine, in addition to the magnetic field, other governing machine equations like electrical and mechanical equations should be brought into calculations simultaneously. Since the machine is supposed to work in the generator mode in this paper, the mechanical equation can be neglected. However, for electrical equations, there are two ways to obtain output voltage and current:

- Calculating the output characteristics by solving differential equations governing the output circuit in the time domain using a numerical or analytical method. In this method, transient response, harmonic content of current and voltage, asymmetrical output current, torque ripple, etc. are considered;

- Using a simple classic model of synchronous machine.

Although the first one is accurate, it is not economical in terms of processing time. Therefore, the latter is used as the performance analysis model of the machine and for optimization process in this work. The procedure initiates by calculating the RMS value of no-load induced voltage, winding inductances, and winding resistance. Winding resistance can be calculated from Eq. (7). From Eqs. (38) to (41), inductances are calculated by assuming a three-phase balanced output current as follows (e.g., for phase $b$ ):

$$
\begin{aligned}
L_{\text {phase }} & =\frac{L_{\text {self }} I_{b}+L_{\text {mutual }} I_{a}+L_{\text {mutual }} I_{c}}{I_{b}} \\
& =L_{\text {self }}-L_{\text {mutual }}=\frac{\lambda_{b b}-\lambda_{a b}}{I_{b}}=\frac{T N_{c}}{3} \frac{\varphi_{b b}-\varphi_{a b}}{I_{b}} \\
& =\frac{T N_{c} l}{I_{b}}\left(\sum_{\substack{n=1,2,4,5,7, \cdots \\
n \neq 3,6, \cdots}}^{\infty} \mathcal{C}_{c o i l, n} \times \sin \left(\frac{n \omega_{w} w_{c g}}{2}\right)\right),
\end{aligned}
$$

where $L_{\text {self }}$ and $L_{\text {mutual }}$ are the self-inductance of each phase and mutual inductance between two different phases, respectively. $\lambda_{b b}$ and $\lambda_{a b}$ are the flux linkage of phase $b$ created by current of phases $b$ and $a$, respectively. RMS value of no-load induced voltage $\left(E_{f}\right)$ due to PM flux and rotor speed is calculated as follows:

$$
\begin{aligned}
E_{f} & =\omega_{s} \frac{\max \left(\lambda_{P M}\right)}{\sqrt{2}}=\frac{\omega_{s} T N_{c}}{3 \sqrt{2}} \max \left(\varphi_{P M}\right) \\
& =\frac{\omega_{s} T N_{c} l}{3 \sqrt{2}} \max \left(A_{1, b}^{P M}-A_{2, b}^{P M}\right) \\
& =\sqrt{2} \frac{\omega_{s} T N_{c} l}{3} \sum_{n=1,3, \cdots}^{\infty} \mathcal{C}_{c o i l, n}^{P M} \sin \left(n \omega_{P M}\left(\frac{w_{c g}}{2}\right)\right)
\end{aligned}
$$

where $\omega_{s}$ is the angular frequency and $\lambda_{P M}$ is the flux linkage of a phase created by PM calculated in Eqs. (34)-(37). Finally, having required parameters at hand, output voltage $\left(V_{t}\right)$ and load current $\left(I_{g}\right)$ for a particular load can be obtained as follows:

$$
\begin{aligned}
I_{g} & =\frac{E_{f}}{j \omega_{s} L_{\text {phase }}+\left(R_{\text {phase }}+R_{\text {load }}\right)}, \\
V_{t} & =E_{f}-\left(j \omega_{s} L_{\text {phase }}+R_{\text {phase }}\right) I_{g},
\end{aligned}
$$

where $R_{\text {load }}$ is the equivalent load resistance and $R_{\text {phase }}$ is calculated in Eq. (7). Input power $\left(P_{i n}\right)$, output power $\left(P_{\text {out }}\right)$, losses $\left(P_{\text {loss }}\right)$, and efficiency $(\eta)$ of the machine can then be obtained as follows:

$$
\begin{aligned}
& P_{\text {in }}=\operatorname{Re}\left(3 E_{f} \overline{I_{g}}\right), \\
& P_{\text {out }}=\operatorname{Re}\left(3 V_{t} \overline{I_{g}}\right), \\
& P_{\text {loss }}=3 R_{\text {phase }}\left|I_{g}\right|^{2}, \\
& \eta=\frac{P_{\text {out }}}{P_{\text {in }}} .
\end{aligned}
$$

Using the developed analytical model and the design considered in Section 2, a 500 Watts machine with approximately 48 Volts output voltage is designed. 
Speed of the rotor based on the average speed of the wind in a specific region is chosen to be 200 RPM. According to what was mentioned in Section 2 about the width of the PMs and coils, independent variables include PM pole pairs, PM thickness, length ratio of two different PMs, air-gap length, outer diameter, ratio of outer to inner diameters, conductor diameter, coil height, and rotor core thickness. One constraint is the outer diameter of the machine, which should be less than $300 \mathrm{~mm}$. As mentioned before, the total cross-section of the PMs is maximum when they have the same length; therefore, $L_{p m 1} / L_{p m 1}=1$. The constraint of the discrete conductor diameter and PM dimension is also considered. The initial design dimensional parameters and output characteristics are shown in Tables 1 and 2, respectively.

As mentioned before, the final purpose of this paper is to have a machine with 500 Watts (for each module) with maximum efficiency, minimum volume of PMs, and minimum weight for the sake of portability. Accordingly, in the next section, the design optimization of the generator is presented.

\section{Design optimization}

Because the simulation time of the developed model is very short, in this section, direct search method is used to optimize the initial design and find the global solution of optimization problem. All the considerations and constraints in the initial design are incorporated in the optimization problem. Among the independent variables, the rotor core thickness has insignificant effect on the output characteristics until the saturation occurs. Moreover, the thickness of rotor core directly affects the iron weight. Therefore, the thickness of the rotor core is considered equal to the initial design. Furthermore, the air gap length is also chosen the same as the initial design due to mechanical considerations. Therefore, among the independent variables, the outer diameter of the machine, number of PMs, height of the PMs, ratio of outer diameter to inner diameter, conductor diameter, and coil thickness are chosen as the optimization variables. The range of variables in the optimization problem is shown in Table 3 .

To maximize the efficiency and minimize the

Table 1. Geometrical parameter of initial and optimal designs.

\begin{tabular}{cccccc}
\hline $\begin{array}{c}\text { Independent } \\
\text { parameters }\end{array}$ & $\begin{array}{c}\text { Initial } \\
\text { design }\end{array}$ & $\begin{array}{c}\text { Final } \\
\text { design }\end{array}$ & $\begin{array}{c}\text { Dependent } \\
\text { parameters }\end{array}$ & $\begin{array}{c}\text { Initial } \\
\text { design }\end{array}$ & $\begin{array}{c}\text { Final } \\
\text { design }\end{array}$ \\
\hline$N_{p m}$ & 20 & 24 & $N_{c}$ & 15 & 18 \\
$H_{p m}(\mathrm{~mm})$ & 8 & 6 & $W_{p m 1}(\mathrm{~mm})$ & 23 & 26 \\
$L_{p m 2} / L_{p m 1}$ & 1 & 1 & $W_{p m 2}(\mathrm{~mm})$ & 33 & 32 \\
$g(\mathrm{~mm})$ & 0.5 & 0.5 & $L_{p m 1}$ & 30 & 25 \\
$D_{o}(\mathrm{~mm})$ & 270 & 300 & $L_{p m 2}$ & 30 & 25 \\
$D_{o} / D_{i}$ & 1.8 & 1.5 & $D_{i}(\mathrm{~mm})$ & 150 & 200 \\
$d_{c u}(\mathrm{~mm})$ & 0.9 & 0.9 & $T_{c}$ & 90 & 66 \\
$H_{c o i l}(\mathrm{~mm})$ & 10.4 & 6.9 & $f_{c u}$ & 0.48 & 0.48 \\
$H_{y}(\mathrm{~mm})$ & 10 & 10 & $W_{c}(\mathrm{~mm})$ & 11.5 & 12.7 \\
\hline
\end{tabular}

Table 2. The performance characteristic of the machine.

\begin{tabular}{|c|c|c|c|c|}
\hline \multirow{2}{*}{ Characteristics } & \multicolumn{2}{|c|}{ Initial design } & \multicolumn{2}{|c|}{ Final design } \\
\hline & 3D FEM & Analytical model & 3D FEM & Analytical model \\
\hline Output power (Watt) & 500 & 500.0 & 500 & 500.0 \\
\hline Rotor speed (rpm) & 200.0 & 200.0 & 200.0 & 200.0 \\
\hline Efficiency (\%) & 86.1 & 86.1 & 88.4 & 88.1 \\
\hline Phase voltage (rms) & 49.5 & 49.1 & 48.4 & 47.4 \\
\hline Line current (rms) & 3.4 & 3.4 & 3.4 & 3.5 \\
\hline Simulation time & $>120 \min$ & $<0.2 \mathrm{~ms}$ & $>90 \min$ & $<0.2 \mathrm{~ms}$ \\
\hline Weight of copper $(\mathrm{kg})$ & \multicolumn{2}{|r|}{1.5} & \multicolumn{2}{|r|}{1.1} \\
\hline Weight of iron (kg) & \multicolumn{2}{|r|}{6.2} & \multicolumn{2}{|r|}{6.2} \\
\hline Weight of PMs (kg) & \multicolumn{2}{|r|}{4.0} & \multicolumn{2}{|r|}{3.1} \\
\hline Total weight (kg) & \multicolumn{2}{|r|}{11.7} & \multicolumn{2}{|r|}{10.4} \\
\hline O.F. & \multicolumn{2}{|r|}{1.00} & \multicolumn{2}{|r|}{0.95} \\
\hline
\end{tabular}




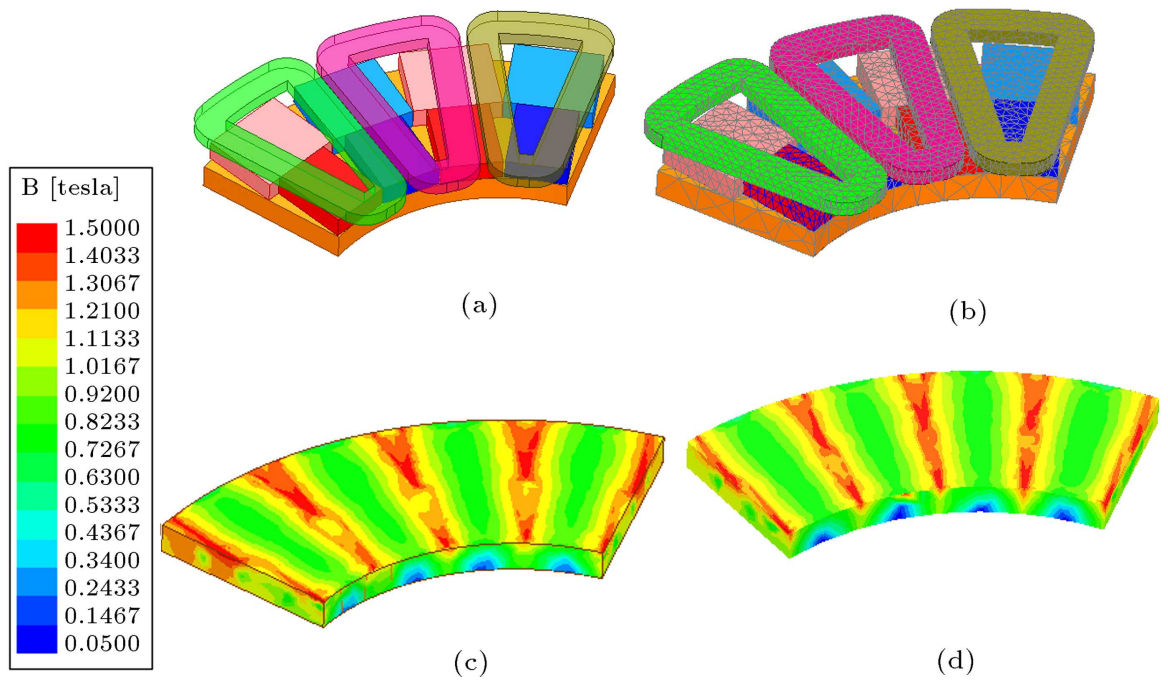

Figure 7. Numerical simulation: (a) The designed generator, (b) schematic of mesh and flux density distribution in rotor core for (c) initial design, and (d) optimal design.

Table 3. Optimization variable and limits.

\begin{tabular}{lcclcc}
\hline \multirow{2}{*}{ Parameters } & \multicolumn{2}{c}{ Limits } & \multirow{2}{*}{ Parameters } & \multicolumn{2}{c}{ Limits } \\
\cline { 2 - 3 } & Min & Max & & Min & Max \\
\hline$D_{o}(\mathrm{~mm})$ & 200 & 300 & $D_{o} / D_{i}$ & 1.5 & 3 \\
$N_{p m}$ & 16 & 24 & $d_{c u}(\mathrm{~mm})$ & 0.6 & 1.2 \\
$H_{p m}(\mathrm{~mm})$ & 5 & 20 & $H_{\text {coil }}(\mathrm{mm})$ & 5 & 15 \\
\hline
\end{tabular}

volume of PMs and total weight of the machine, the objective function is considered as in the following by normalizing the efficiency, PMs, and copper and iron weights by using initial design value and appropriate coefficients:

$$
\begin{aligned}
\text { O.F. }= & 0.85 \times \frac{86.1}{\eta}+0.08 \times \frac{\text { PMs weight }}{4.0}+0.04 \\
& \times \frac{\text { Copper weight }}{1.5}+0.03 \times \frac{\text { Iron weight }}{6.2} .
\end{aligned}
$$

By searching throughout approximately 2.0 million cases using the developed analytical model, the one with minimum objective function is selected as the optimum design. Geometrical parameters and output characteristics of optimal design are shown in Tables 1 and 2 , respectively.

As demonstrated earlier, after optimization, the following outcome is expected: PM volume is reduced by $22.5 \%$; efficiency is increased by $2.3 \%$; copper volume is decreased by $26.7 \%$; and total iron weight does not change. Finally, the whole weight of the machine is reduced by $11.1 \%$.

It is worth mentioning that the whole optimization process takes less than 15 minutes due to a very short simulation time at each iteration (less than $0.2 \mathrm{~ms})$.

\section{Time stepping finite element validation}

To evaluate the accuracy of the developed model and optimization process, their results are compared with those of 3D TSFEM. Figure 7(a) shows the 3D structure of the designed generator in ANSYS Maxwell software. To shorten the FEA simulation time, periodic and symmetry boundary conditions are used. Figure 7(b) shows the schematic of the mesh on the studied generator. Flux density distribution on the rotor core for initial and optimal designs is shown in Figure 7(c) and (d), respectively. Figure 8(a) and (b) show the magnetic flux lines in the cross-section of the machine at a specific radius for both designs. The back EMF of the three-phase winding is obtained from 3D TSFEM for the initial and final designs, as shown in Figure 9. Furthermore, the output characteristics of designed machines are shown in Table 2. It can be seen that the calculation error of the analytical model with respect to the FEM simulation is less than $3 \%$. Whereas the required time for FEM analysis is longer than $90 \mathrm{~min}$, this time for the analytical model is less than 0.2 milliseconds with the same system to run the process. Fast calculation time for the proposed hybrid model with a minimum amount of compromise in accuracy of the model makes it a perfect basis to run the optimization process.

\section{Conclusion}

In this paper, design optimization of a coreless Axial Flux Permanent Magnet (AFPM) generator was presented. In this regard, a fast and accurate hybrid analytical model by combining the solution of Maxwell equations in different regions with the classical model of the synchronous generator was developed first. Then, 


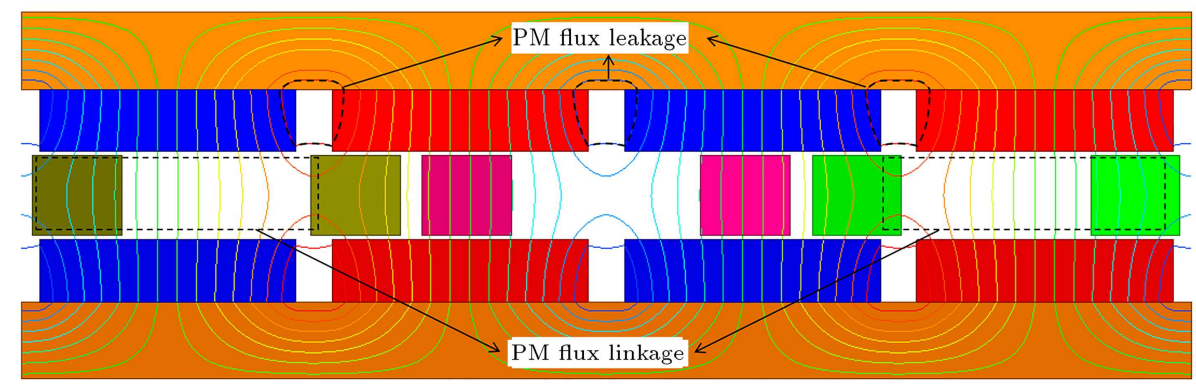

(a)

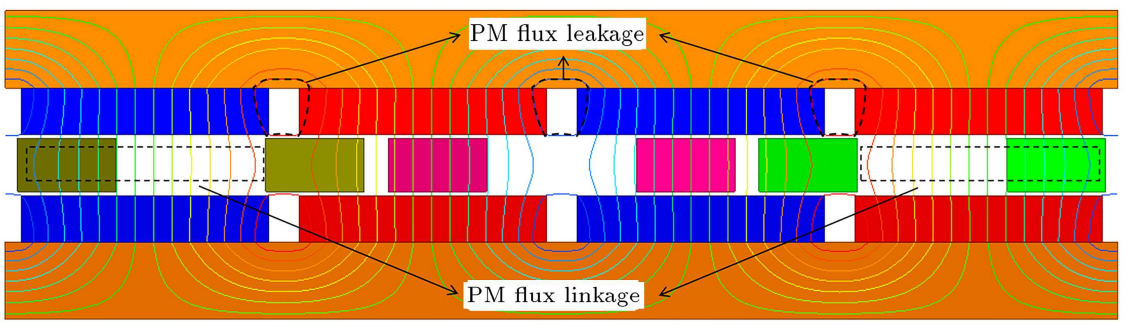

(b)

Figure 8. Magnetic flux lines at a specific radius of AFPM generator obtained using 2D FEA for (a) initially designed generator and (b) optimally designed generator.

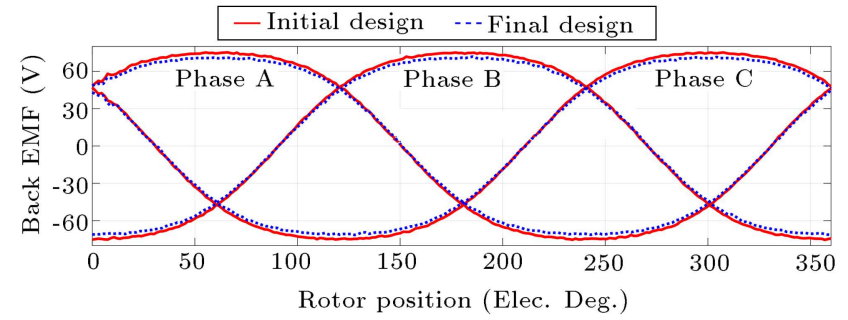

Figure 9. Back EMF of initial and final designs obtained from 3D TSFEA.

the developed model was used to design a 500 Watts generator. Afterwards, the initial design was optimized using a direct search optimization method. The optimization process resulted in reducing the weight of the machine by $11.1 \%$ (from $11.7 \mathrm{~kg}$ to $10.4 \mathrm{~kg}$ ) and increasing the efficiency from 86.1 to 88.4. By comparing the results of the proposed analytical model and 3D TSFEA, the calculation error was seen to be less than $3 \%$. Furthermore, the simulation time of the model was shorter than $0.2 \mathrm{~ms}$, while the simulation time of the FEA was longer than 90 min.

\section{Acknowledgments}

The authors gratefully thank Enercrop Co. and Eng. M. Ebrahimzadeh for their support and permission to publish this work.

\section{References}

1. Blaabjerg, F., Ma, K., and Yang, Y. "Power electronics-The key technology for Renewable energy systems", Int. Conf. on Ecological Vehicles and Renewable Energies, Monte-Carlo, pp. 1-11 (2014).

2. Ghulam, A. and Uzma, A. "Design, construction and study of small scale vertical axis wind turbine based on a magnetically levitated axial flux permanent magnet generator", Renew. Energ., 101, pp. 286-292 (2017).

3. Kashani, S.A.A. "Optimal design and analysis of a novel reluctance axial flux magnetic gear", Sci. Iran., 29(3), pp. 1573-1580 (2022).

4. Saadat, J. and Ardebili, M. "Cogging torque reduction in axial-flux permanent magnet wind generators with yokeless and segmented armature by radially segmented and peripherally shifted magnet pieces", Renew. Energ., 99, pp. 95-106 (2016).

5. Chen, Y., Pillay, P., and Khan, A. "PM wind generator topologies", IEEE Trans. Ind. Appl., 41(6), pp. 16191626 (2005).

6. Abbaszadeh, K. and Rahimi, A. "Analytical quasi $3 \mathrm{D}$ modeling of an axial flux PM motor with static eccentricity fault", Sci. Iran., 22(6), pp. 2482-2491 (2015).

7. Ho, S.-L., Niu, S., and Fu, W.-N. "Design and analysis of a novel axial-flux electric machine", IEEE Trans. Magn., 47(10), pp. 4368-4371 (2011).

8. Mahmoudi, A., Kahourzade, S., Rahim, N.-A., et al. "Design, analysis, and prototyping of an axial-flux permanent magnet motor based on genetic algorithm and finite-element analysis", IEEE Trans. Magn., 49(4), pp. 1479-1492 (2013).

9. RamaRao, K.S., Sunderan, T., and Ref'atAdiris, M. "Performance and design optimization of two model 
based wave energy permanent magnet linear generators", Renew. Energ., 101, pp. 196-203 (2017).

10. Wei, L., Nakamura, T., and Imai, K. "Development and optimization of low-speed and high-efficiency permanent magnet generator for micro hydro-electrical generation system", Renew. Energ., 147(1), pp. 16531662 (2020).

11. Wang, X., Zhao, M., Tang, L., et al. "Fault-tolerant analysis and design of AFPMSM with multi-disc type coreless open-end winding", IEEE Access, 8, pp. 171744-171753 (2020).

12. Arand, S.-J. and Ardebili, M. "Multi-objective design and prototyping of a low cogging torque axial-flux PM generator with segmented stator for small-scale directdrive wind turbines", IET Elect. Power Appl., 10(9), pp. 889-899 (2016).

13. Minaz, M.-R. and Çelebi, M. "Design and analysis of a new axial flux coreless PMSG with three rotors and double stators", Results Phys., 7, pp. 183-188 (2017).

14. Hijazi, T.-M., El-Masri, B., and Arkadan, A.-A. "Design evaluation of conventional and toothless stator wind power axial-flux PM generator", IEEE Conf. on Electromagnetic Field Computation, Miami, FL, pp. 1-1 (2016). DOI: 10.1109/CEFC.2016.7815875

15. Ishikawa, T., Amada, S., Segawa, K., et al. "Proposal of a radial- and axial-flux permanent-magnet synchronous generator", IEEE Trans. Magn., 53(6), pp. 1-4 (2017).

16. Wu, D., Yang, J., Chan, T.-F., et al "Axial-flux permanent-magnet synchronous generator with coreless armature and non-integral coil-pole ratio", IET Renew. Power Gener., 13(2), pp. 245-252 (2019).

17. Naderi, P., Sharouni, S., and Moradzadeh, M. "Analysis of partitioned stator flux-switching permanent magnet machine by magnetic equivalent circuit", Int. J. Elect. Power Energy Syst., 111, pp. 369-381 (2019).

18. Widyan, M.S. and Hanitsch, R.E. "High-power density radial-flux permanent-magnet sinusoidal three-phase three-slot four-pole electrical generator", Int. J. Elect. Power Energy Syst., 43, pp. 1221-1227 (2012).

19. Wen, Z., Xiong, B., and Gu, G. "Optimization design of low speed axial flux halbach permanent-magnet generator with PCB winding", Int. Conf. on Electrical Machines and Systems Harbin, China, pp. 1-4 (2019).

20. Nasiri-Gheidari, Z. and Lesani, H. "Optimal design of adjustable air-gap, two-speed, capacitor-run, singlephase axial flux induction motors", IEEE Trans. Energy Convers., 28(3), pp. 543-552 (2013).

21. Daniar, A., Nasiri-Gheidari, Z., and Tootoonchian, F. "Performance analysis of linear variable reluctance resolvers based on an improved winding function approach", IEEE Trans. Energy Convers., 33(3), pp. 1422-1430 (2018).

22. Radwan-Praglowska, N., Borkowski, D., and Wegiel, T. "Model of coreless axial flux permanent magnet generator", International Symposium on Electrical Machines (SME), Naleczow, pp. 1-6 (2017).
23. Saneie, H. and Nasiri-Gheidari, Z. "Performance analysis of outer-rotor single-phase induction motor based on Magnetic Equivalent Circuit (MEC)", IEEE Trans. Ind. Electron., 68(2), pp. 1046-1054 (2021).

24. Gysen, B.-L.-J., Meessen, K.-J., Paulides, J.-J.-H., et al. "General formulation of the electromagnetic field distribution in machines and devices using Fourier analysis", IEEE Trans. Magn., 46(1), pp. 39-52 (2010).

25. Daghigh, A., Javadi, H., and Javadi, A. "Improved analytical modeling of permanent magnet leakage flux in design of the coreless axial flux permanent magnet generatorx", Can. J. Electr. Comput. Eng., 40(1), pp. 3-11 (2017).

26. Alipour-Sarabi, R., Nasiri-Gheidari, Z., and Oraee, H. "Development of a 3-D magnetic equivalent circuit model for axial flux machines", IEEE Trans. Ind. Electron., 67(7), pp. 5758-5767 (2019).

27. Zhu, J., Li, S., Song, D., et al. "Multi-objective optimisation design of air-cored axial flux PM generator", IET Electr. Power Appl., 12(9), pp. 1390-1395 (2018).

28. Raabe, N. "An algorithm for the filling factor calculation of electrical machines standard slots", Int. Conf. on Electrical Machines, Berlin, pp. 981-986 (2014).

29. Jalali, P., Boroujeni, S.-T., and Bianchi, N. "Analytical modeling of slotless eccentric surface-mounted PM machines using a con-formal transformation", IEEE Trans. Energy Convers., 32(2), pp. 658-666 (2017).

\section{Biographies}

Hamid Saneie (Student member, IEEE) received the BSc degree from Shahr-e Kord University, Shahr-e Kord, Iran in 2015 and MSc degree from the Sharif University of Technology, Tehran, Iran in 2017, where he is currently working toward the $\mathrm{PhD}$ degree, all in Electrical Engineering. His research interests include modeling, design, optimization, and performance analysis of electrical machines and electromagnetic sensors.

Ahmad Daniar (Student member, IEEE) received the BSc degree in Electrical Engineering from the University of Tabriz, Tabriz, Iran in 2014 and the MSc degree in the same field from Sharif University of Technology, Tehran, Iran in 2017. He is currently working toward the $\mathrm{PhD}$ degree in Electrical Engineering at the University of Texas at Dallas, Richardson, TX, USA. His research interests include design, analysis and fault diagnosis of electric machines and electromagnetic components, drive and control of electric machines, and power electronics converters.

Zahra Nasiri-Gheidari (Senior member, IEEE) received the BSc degree from the Iran University of 
Sciences and Technology, Tehran, Iran in 2004 and the MSc and PhD degrees from University of Tehran, Tehran in 2006 and 2012, respectively, all in Electrical Engineering. She is currently an Associate Professor at the Department of Electrical Engineering, Sharif University of Technology. Her research interests include design, optimization, and performance analysis of electrical machines and electromagnetic sensors. 